\title{
Modeling of water and salt transfer in the initial period of plant development
}

\author{
Aliakbar Khojiyev ${ }^{1 *}$, Rustam Muradov ${ }^{1}$, Shakhnoza Khojiyeva $^{2}$, Khurshida Yakubova $^{1}$ \\ ${ }^{1}$ Tashkent institute of irrigation and agricultural mechanization engineers, Tashkent, Uzbekistan \\ ${ }^{2}$ Tashkent pharmaceutical institute, Tashkent, Uzbekistan
}

\begin{abstract}
Water - as moisture, has an essential role in all biochemical processes of plants; all vital processes occurring in a vegetative organism can proceed normally only under the condition of sufficient saturation of cages by moisture. Results of theoretical researches on dynamics of ground humidity have shown: (1) similarity of physical processes of change of humidity of soil on different irrigated areas (2) hysteresis of the nature of humidity at irrigation and drainage. (3) sharp recession of humidity of soil in the root zone.
\end{abstract}

\section{Introduction}

Among the main factors in the arid zone, an important role is played by the water and thermal regimes of soils, which mainly determine the fate of the crop of irrigated crops. This is explained by the fact that the subsoil processes are closely related to weather conditions. Depending on their behavior, the need for appropriate ameliorative impacts on the agricultural field is established[14, 16, 17, 19, 20].

At this stage, it is necessary to use mathematical models for soils most common in the region with the aim of reclamation. Such models are important as a basis for optimizing the use of land resources in irrigated areas by changing the structure of land use, specializing in agriculture, etc. With these studies, it is necessary to use the achievements of the fundamental sciences, the mathematical apparatus, and the computer. The introduction of new methods in land reclamation is a slow and time-consuming process, because soils must be considered as a multiparameter and dynamically changing object.

The conducted studies to date have proved the inconsistency of the interpretations of the management of the productivity of agroecosystems, when only a few isolated indicators were taken into account or the informativeness of the integral indicators was usually judged from the data of correlation and regression analyzes that do not always reflect the actual processes taking place in the soil- plant". In the methodology for assessing soils as an object of intensive agricultural use, a new stage has come-the transition from bathing assessments, studies of individual optimal parameters to the analysis of the productivity of agroecosystems based on their mathematical modeling $[1,2,5,6,11,12,15,18]$.

\footnotetext{
${ }^{1 *}$ Corresponding author: aliakbar-x@mail.ru
} 


\section{Methods}

The spatial-temporal dynamics of soil moisture were investigated in several Water Consumers Association.

On the demonstration sites, cotton was grown; space between the rows was $90 \mathrm{~cm}$. Five sampling sites (four under cotton grown area and one at non-vegetated area - control) with four replications of each were selected randomly. Soil samples were collected annually during 2017-2018.

The experiment consists of two parts: The first is the analysis of soil moisture dynamics based on the irrigation frequency. The soil moisture was measured right before and after the irrigation; the next was as well as 1, 2, 3 and 5 days before and after the irrigation. The sampling was replicated four times. Moisture is determined from $10-\mathrm{cm}$ layers and in the root and top soil - from 0.5 and 5-10 cm layers. The sampling arrangement is shown in Fig. 1.

In the initial period of plant development under steady-state conditions, when transpiration of Em can be neglected, the following mathematical model will be used for a two-layer medium consisting of arable and subarable layers $[1,2,7,8,9,10]$.

With soluble salts and small content in the solid phase (for example, chlorine), the equation of salt transfer satisfactorily describes the distribution of salts observed in nature and experiments without the last term $\gamma\left(c_{s}-c\right)$, i.e.:

We note that in this case, D takes into account the peculiarities of the motion of solutions in a nonsolvent medium (the so-called longitudinal and transverse effects) and is not equal to the usual diffusion coefficient in a resting solution $[1,2,3,5]$.

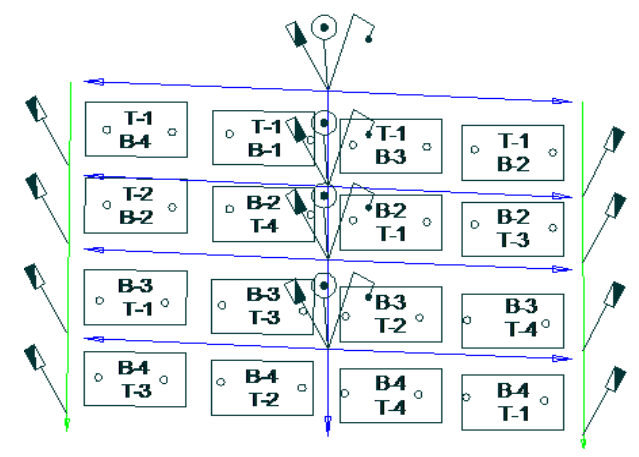

Fig-1. Experimental schemes for research sites.
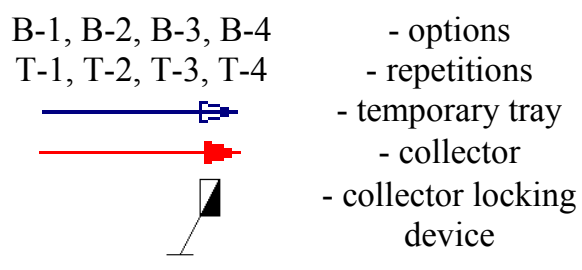

$$
\left\{\begin{array}{c}
0 \leq z \leq z_{1} \\
\frac{d}{d z}\left[D_{1}\left(W_{1}\right) \frac{d W_{1}}{d z}\right]-\frac{d K_{1}\left(W_{1}\right)}{d z}=0, \\
\frac{d}{d z}\left[D_{N_{1}}\left(W_{1}\right) \frac{d N_{1}\left(W_{1}\right)}{d z}\right]-\frac{d V_{N_{1}}\left(W_{1}\right)}{d z}=0, \\
z_{1} \leq z \leq L \\
\frac{d}{d z}\left[D_{2}\left(W_{2}\right) \frac{d W_{2}}{d z}\right]-\frac{d K_{2}\left(W_{2}\right)}{d z}=0, \\
\frac{d}{d z}\left[D_{N_{2}}\left(W_{2}\right) \frac{d N_{2}\left(W_{2}\right)}{d z}\right]-\frac{d V_{N_{2}}\left(W_{2}\right)}{d z}=0
\end{array}\right.
$$




$$
\begin{aligned}
& W_{1}(0)=W_{I M}=\text { const } \\
& N_{1}(0)=N_{I M}=\text { const } \\
& W_{1}\left(Z_{1}\right)=W_{2}\left(Z_{1}\right) \\
& N_{1}\left(Z_{1}\right)=N_{2}\left(Z_{1}\right) \\
& \quad\left[K_{1}\left(W_{1}\right)-D_{1}\left(W_{1}\right) \frac{d W_{1}}{d z}\right]_{Z=Z_{1}}=\left[K_{2}\left(W_{2}\right)-D_{2}\left(W_{2}\right) \frac{d W_{2}}{d z}\right]_{Z=Z_{1}}(6) \\
& V_{N_{1}}\left(W_{1}\right)-\left.D_{N_{1}}\left(W_{1}\right) \frac{d N_{1}\left(W_{1}\right)}{d z}\right|_{Z=Z_{1}}=V_{N_{2}}\left(W_{2}\right)-\left.D_{N_{2}}\left(W_{2}\right) \frac{d N_{2}\left(W_{2}\right)}{d z}\right|_{Z=Z_{1}} \\
& W_{2}(L)=W_{M C}, \\
& N_{2}(L)=N_{M C}
\end{aligned}
$$

where the following designations are entered for the arable and sub-plow layers, respectively: $\mathrm{W}_{1}, \mathrm{~W}_{2}$ are volumetric humidity; coefficients of moisture conductivity are adopted in the form $[1 ; 4]$ :

$$
K_{1}\left(W_{1}\right)=A_{1} e^{A_{2} z}, K_{2}\left(W_{2}\right)=B_{1} e^{B_{2} z} .
$$

the speed of water movement in the ground is taken as [1]:

$$
V_{N_{1}}=R_{1} e^{R_{2} Z}, \quad V_{N_{2}}=P_{1} e^{P_{2} Z}
$$

Because the stationary regime is considered for the diffusivity coefficients, their mean values

$$
\begin{aligned}
& D_{1}\left(W_{1}\right)=D_{1}=\text { const, } \quad D_{2}\left(W_{2}\right)=D_{2}=\text { const } \\
& D_{N_{1}}\left(W_{1}\right)=D_{N_{1}}=\text { const, } \quad D_{N_{2}}\left(W_{2}\right)=D_{N_{2}}=\text { const },
\end{aligned}
$$

where: $\mathrm{L}$ is groundwater depth, $\mathrm{m}$;

$\mathrm{Z}_{1}$ is boundary between arable and subsoil layers, $\mathrm{m}$;

$\mathrm{W}_{I M}$ is some intermediate moisture capacity between wilting moisture $\mathrm{W}_{3}$ and the maximum moisture capacity $\mathrm{W}_{M M C}$, T.e.

$$
W_{3}<W_{I M}<W_{M M C}
$$

where: $\mathrm{W}_{M C}$ is full moisture capacity;

$\mathrm{Z}$ is vertical coordinate directed down from the earth's surface.

Also, $\mathrm{N}_{I M}$ is an intermediate concentration of the salts between the concentration of the salts in the wash water $\mathrm{N}_{\mathrm{W}}$ and the concentration of the limiting saturation of water $\mathrm{N}_{\mathrm{S}}$, i.e.

$$
N_{W} \leq N_{I M} \leq N_{S}
$$


As a result of these notations, we rewrite the boundary value problem (1) - (9) as follows

$$
\begin{aligned}
& \left\{\begin{array}{l}
D_{1} \frac{d^{2} W_{1}}{d z^{2}}-A_{1} A_{2} e^{A_{2} Z}=0 \\
D_{N_{1}} \frac{d^{2} N_{1}}{d z^{2}}-R_{1} R_{2} e^{R_{2} Z}=0 \\
D_{2} \frac{d^{2} W_{2}}{d z^{2}}-B_{1} B_{2} e^{B_{2} Z}=0 \\
D_{N_{2}} \frac{d^{2} N_{2}}{d z^{2}}-P_{1} P_{2} e^{P_{2} Z}=0
\end{array}\right. \\
& A_{1} e^{A_{2} Z}-\left.D_{1} \frac{d W_{1}}{d z}\right|_{Z=Z_{1}}=B_{1} e^{B_{2} Z}-\left.D_{2} \frac{d W_{2}}{d z}\right|_{Z=Z_{1}}, \\
& R_{1} e^{R_{2} Z}-\left.D_{N_{1}} \frac{d N_{1}}{d z}\right|_{Z=Z_{1}}=P_{1} e^{P_{2} Z}-\left.D_{N_{2}} \frac{d N_{2}}{d z}\right|_{Z=Z_{1}}
\end{aligned}
$$

where $A_{1}, A_{2}, B_{1}, B_{2}, D_{1}, D_{2}, R_{1}, R_{2}, P_{1}, P_{2}, D_{N 1}, D_{N 2}$ are some constants determined by comparing the analytical solution with the experimental data [1];

Integrating the first equation of system (16), we will successively find

$$
\begin{gathered}
\frac{d^{2} W_{1}}{d z^{2}}-\frac{A_{1} A_{2}}{D_{1}} e^{A_{2} Z}=0 \\
\frac{d W_{1}}{d z}=\frac{A_{1}}{D_{1}} e^{A_{2} Z}+C_{1} \\
W_{1}=\frac{A_{1}}{A_{2} D_{1}} e^{A_{2} Z}+C_{1} z+C_{2}
\end{gathered}
$$

Similarly, after integrating the other equations of the same system, we obtain

$$
\begin{gathered}
N_{1}=\frac{R_{1}}{R_{2} D_{N_{1}}} e^{R_{2} Z}+C_{3} z+C_{4}, \\
W_{2}=\frac{B_{1}}{B_{2} D_{2}} e^{B_{2} Z}+C_{5} z+C_{6} \\
N_{2}=\frac{P_{1}}{P_{2} D_{N_{2}}} e^{P_{2} Z}+C_{7} z+C_{8}
\end{gathered}
$$


Using condition (2), we find from (19)

$$
C_{2}=W_{I M}-\frac{A_{1}}{A_{2} D_{1}}
$$

We also determine C4 from the conditions (3) and (26)

$$
C_{4}=N_{I M}-\frac{R_{1}}{R_{2} D_{N 1}}
$$

Based on (6) and (7), we find the relation

$$
\begin{gathered}
C_{5}=C_{1} \frac{D_{2}}{D_{1}} \\
C_{7}=C_{3} \frac{D_{N_{2}}}{D_{N_{1}}}
\end{gathered}
$$

Expressions (8) and (9), using (8) and (9), we obtain

$$
\begin{aligned}
& W_{M C}=\frac{B_{1}}{B_{2} D_{2}} e^{B_{2} L}+C_{5} L+C_{6} \\
& N_{M C}=\frac{P_{1}}{P_{2} D_{N_{2}}} e^{P_{2} L}+C_{3} \frac{D_{N_{2}}}{D_{N_{1}}} L+C_{8}
\end{aligned}
$$

The dependence of $\mathrm{C} 6$ on $\mathrm{Cl}$ is found from (24) with allowance for (22)

$$
C_{6}=W_{M C}-\frac{B_{1}}{B_{2} D_{2}} e^{B_{2} L}-C_{1} \frac{D_{2}}{D_{1}} L
$$

The value of C8 is determined from (19) with allowance for (23)

$$
C_{8}=N_{M C}-\frac{P_{1}}{P_{2} D_{N_{2}}} e^{P_{2} L}-C_{3} \frac{D_{N_{2}}}{D_{N_{1}}} L
$$

\section{Results and Discussion}

Relation (4) with allowance for (21), (22), and (26) allows us to determine C1 from equality

$$
W_{M C}-\frac{B_{1}}{B_{2} D_{2}}\left[e^{B_{2} L}-e^{B_{2} Z_{1}}\right]-C_{1} \frac{D_{2}}{D_{1}}\left[L-Z_{1}\right]=W_{I M}-\frac{A_{1}}{A_{2} D_{1}}\left[e^{A_{2} Z_{1}}-1\right]+C_{1} Z_{1}
$$

From where we find 


$$
C_{1}=\frac{W_{M C}-W_{I M}-\frac{A_{1}}{A_{2} D_{1}}\left[e^{A_{2} Z_{1}}-1\right]-\frac{B_{1}}{B_{2} D_{2}}\left[e^{B_{2} L}-e^{B_{2} Z_{1}}\right]}{\frac{D_{2}}{D_{1}}\left[L-Z_{1}\right]+Z_{1}},
$$

It is possible to establish the value of C3 from equation (22), (23), and (27)

$$
N_{M C}-\frac{P_{1}}{P_{2} D_{N_{2}}}\left[e^{P_{2} L}-e^{P_{2} Z_{1}}\right]-C_{3} \frac{D_{N_{2}}}{D_{N_{1}}}\left[L-Z_{1}\right]=N_{\Pi P}-\frac{R_{1}}{R_{2} D_{N_{1}}}\left[e^{R_{2} Z_{1}}-1\right]+C_{3} Z_{1}
$$

Hence we find

$$
C_{3}=\frac{N_{M C}-N_{I M}-\frac{R_{1}}{R_{2} D_{N_{1}}}\left[e^{R_{2} Z_{1}}-1\right]-\frac{P_{1}}{P_{2} D_{N_{2}}}\left[e^{P_{2} L}-e^{P_{2} Z_{1}}\right]}{\frac{D_{N_{2}}}{D_{N_{1}}}\left[L-Z_{1}\right]+Z_{1}},
$$

Substituting the values of arbitrary $C_{1}, C_{2}, C_{3}$ and $C_{4}$ in (19) and (20), we obtain the distribution of volumetric moisture and salt concentration in the arable layer as a function of $\mathrm{z}$.

$$
\begin{gathered}
W_{1}=W_{I M}+\frac{A_{1}}{A_{2} D_{1}}\left[e^{A_{2} Z}-1\right]+\left[\frac{W_{M C}-W_{I M}-\frac{A_{1}}{A_{2} D_{1}}\left[e^{A_{2} Z_{1}}-1\right]-\frac{B_{1}}{B_{2} D_{2}}\left[e^{B_{2} L}-e^{B_{2} Z_{1}}\right]}{\frac{D_{2}}{D_{1}}\left[L-Z_{1}\right]+Z_{1}}\right] z \\
N_{1}=N_{I M}+\frac{R_{1}}{R_{2} D_{N_{1}}}\left[e^{R_{2} Z}-1\right]+\left[\frac{N_{M C}-N_{I M}-\frac{R_{1}}{R_{2} D_{N_{1}}}\left[e^{R_{2} Z_{1}}-1\right]-\frac{P_{1}}{P_{2} D_{N_{2}}}\left[e^{P_{2} L}-e^{P_{2} Z_{1}}\right]}{\frac{D_{N_{2}}}{D_{N_{1}}}\left[L-Z_{1}\right]+Z_{1}}\right] z \\
0 \leq \mathrm{Z} \leq \mathrm{Z}_{1}
\end{gathered}
$$

The definite values of the constants $\mathrm{C}_{5} \mathrm{C}_{6}, \mathrm{C} 7$, and $\mathrm{C}_{8}$ in (21) and (22) yield the distribution of the volumetric moisture content and the salt concentration in the subpolar layer as a function of $\mathrm{z}$.

$$
\begin{gathered}
W_{2}=W_{M C}-\frac{B_{1}}{B_{2} D_{2}}\left(e^{B_{2} L}-e^{B_{2} Z}\right)-\left(\frac{W_{M C}-W_{I M}-\frac{A_{1}}{A_{2} D_{1}}\left[e^{A_{2} Z_{1}}-1\right]-\frac{B_{1}}{B_{2} D_{2}}\left[e^{B_{2} L}-e^{B_{2} Z_{1}}\right]}{\left[L-Z_{1}\right]+Z_{1}}\right)(L-z) \\
N_{2}=N_{M C}-\frac{P_{1}}{P_{2} D_{N_{2}}}\left(e^{P_{2} L}-e^{P_{2} Z}\right)-\left(\frac{N_{M C}-N_{I M}-\frac{R_{1}}{R_{2} D_{N_{1}}}\left[e^{R_{2} Z_{1}}-1\right]-\frac{P_{1}}{P_{2} D_{N_{2}}}\left[e^{P_{2} L}-e^{P_{2} Z_{1}}\right]}{\left[L-Z_{1}\right]+Z_{1}}\right)(L-z) \\
Z_{1} \leq \mathrm{Z} \leq \mathrm{L}
\end{gathered}
$$




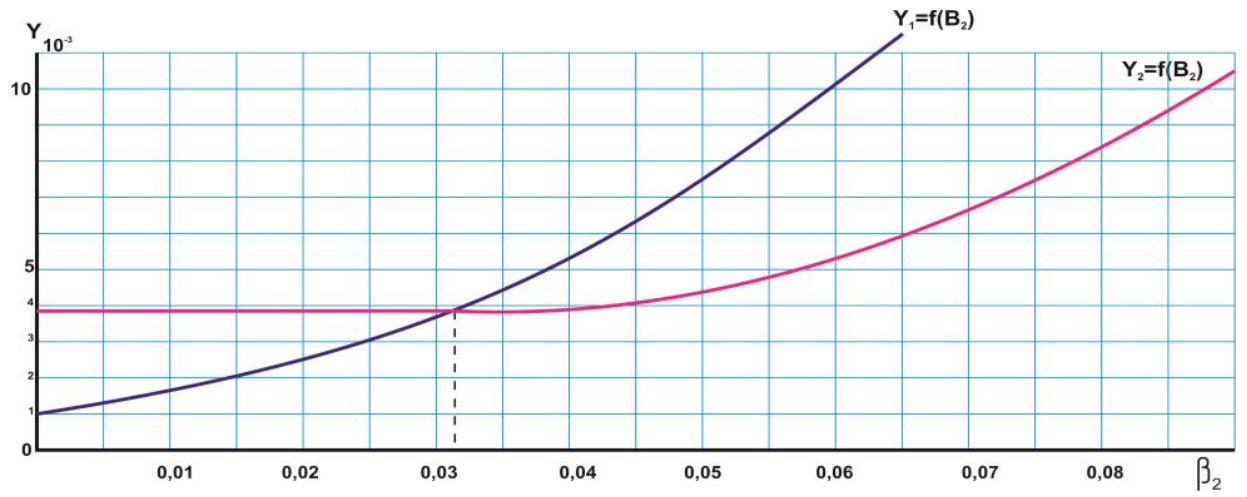

Fig 2. Determination of the value of $\beta 2$ by the transcendental equation.

The change in moisture content and concentration of salts at various initial surface moisture indices without considering the development of the plant root system for the conditions of the WUA "Uzbekistan" in the Syrdarya region is shown in Fig. 2. Determination of the constants was carried out according to the acad. F.B. Abutaliev given in [1, 2]. Fig. 2. shows the change in soil moisture during the initial period of plant development (winter wheat). The bend point on the graph indicates the boundary between the arable and subplow layers $(42 \mathrm{~cm})$.

\section{Conclusions}

1. The developed models (33) - (34) can be used to calculate moisture and salt transfer both in the initial period of plant development and in the calculation of washing of saline lands.

2. The use of models and the coefficients of the mathematical model to determine the parameters of moisture and salt transfer make it possible to calculate the reserve of soil moisture and optimize the sowing time at its maximum value.

\section{References}

1. Khojiev A., Muradov R. Moisture and salt transfer in the initial period of plant development. The path of science. International Journal, No. 8 (54), pp. 50-56. (2018).

2. Khojiyev A., Muradov R., Khaydarov T., Rajabov N., Utepov B. Some results of moisture and salt transfer in the initial period of plant development. International Journal of Engineering and Advanced Technology (IJEAT)/ Volume-9, pp 69076911. India, (2019).

3. Muradov R., Khojiev A. The optimal solution of leaching rates with a deficit of irrigation water. Agro Ilm Magazine, No. 5 (49), pp. 83-84. (2017).

4. Khojiyev A., Khaydarov T., Rajabov N., J.Pulatov. Optimal solution leaching rates with a deficit of irrigation water. IOP Conf. Series: Materials Science and Engineering 883 (2020) 012091, CONMECHYDRO - (2020).

5. Khojiyev A., Muradov R.A., Khaydarov T., J.Pulatov. Changes in the exchange of salt and moisture in groundwater management. IOP Conf. Series: Materials Science and Engineering 883 (2020) 012091, CONMECHYDRO - (2020).

6. Khojiyev A., Muradov R. Moisture and salt transfer in the initial period of plant 
development. The Way of Science. International scientific journal.Volgograd, № 8 (54) pp.50-54 (№5-Global Impact Factor. IF-0.543). Russia. (2018).

7. Z. Mirkhasilova, L. Irmuhamedova, S. Kasymbetova, G. Akhmedjanova M. Mirkhosilova. Rational use of collector-drainage water 2020 IOP Conf. Ser.: Mater. Sci. Eng. 883 012092. CONMECHYDRO (2020).

8. Khamidov M.X., Suvanov B.U. Irrigation of crops by subirrigation//Monograph. Publishing house "UZBEKISTAN". pp.155 Tashkent. (2018).

9. Khamidov M.X., Isaev S.X. and others. The effect of subirrigation of cotton on hydromorphic soils on cotton yield // Journal of Irrigation and Land Reclamation. №2. pp.5 Tashkent, (2015)

10. Matyakubov B. How efficient irrigation can ensure water supply in the Lower Amudarya basin of Uzbekistan, International Water and Irrigation, 23 (3), pp. 2627. (2003)

11. Hamidov, A., Khamidov, M., Ishchanov, J. Impact of climate change on groundwater management in the northwestern part of Uzbekistan.Agronomy, 10(8), 1173. (2020).

12. Khamidov, M.K., Khamraev, K.S., Isabaev, K.T. Innovative soil leaching technology: A case study from Bukhara region of Uzbekistan. IOP Conference Series: Earth and Environmental Science, 422(1), 012118. (2020).

13. Khamidov, M., Khamraev, K., Azizov, S., Akhmedjanova, G. Water saving technology for leaching salinity of irrigated lands: A case study from Bukhara region of Uzbekistan. Journal of Critical Reviews, 7(1), pp.499-509. (2020).

14. Mirzoolim Avliyakulov, Normat Durdiev, Nurmamat Rajabov, Farruhjon Gopporov, Adkham Mamataliev. The changes of cotton seed-lint yield in parts of furrow length under different irrigation scheduling. Journal of Critical Reviews ISSN- 2394-5125 Vol 7, Issue 5, pp 838-843. (2020).

15. Saidhujaeva Nafisa, Nulloev Ulugbek, Mirkhasilova Zulfiya, Mirnigmatov Botir, Irmukhamedova Ludmila Production of Plant Product as a Process of Functioning Biotechnical System. IJEAT. ISSN: 2249-8958, Volume-9 Issue-1, October (2019).

16. Mirkhasilova Z.K. Ways to improve the water availability of irrigated lands. European science review No. pp.7-8. july-august. (2018).

17. Z. Mirkhasilova, L.Irmuhamedova G.Akhmedjanova, D. Tursunova. Transfer of vegetable water flows for full support of drained water from wells of vertical drainage. International journal of advanced research in science, engineering and technology. ISSN:2350-0328. Vol.6, Issue 5, pp 9424-94262. May (2019).

18. Khamidov M., Matyakubov B., Isabaev K. "Substantiation of cotton irrigation regime on meadow-alluvial soils of the Khorezm oasis" // Journal of Critical Reviews, ISSN- 2394-5125, Volume 7, Issue 4, - pp. 347 - 353. (2020),

19. Matyakubov B., Begmatov I., Raimova I., Teplova G. "Factors for the efficient use of water distribution facilities"// CONMECHYDRO - (2020), IOP Conf. Series: Materials Science and Engineering 883 (2020) 012050 doi:10.1088/1757899X/883/1/012050.

20. Matyakubov B., Mamazhonov M., Teplova G, Shakirov B., Shakirov B. "Forebays of the poligonal cross - section of the irrigating pumping station"// CONMECHYDRO - (2020), IOP Conf. Series: Materials Science and Engineering 883 (2020) 012050 doi:10.1088/1757-899X/883/1/012050. 